SELECTED PAPER AT NCSP'20

\title{
Multipath Content Acquisition Method Using Overhearing in ICN
}

\author{
Kaede Shinohara and Shigeki Shiokawa \\ Information and Computer Sciences, Kanagawa Institute of Technology \\ 1030 Shimo-ogino, Atsugi, Kanagawa 243-0292, Japan \\ E-mail: s2085004@cce.kanagawa-it.ac.jp, shiokawa@nw.kanagawa-it.ac.jp
}

\begin{abstract}
Recently, ICN (Information-Centric Networking) has been applied to wireless environments such as mobile ad hoc networks (MANETs). In a MANET, each node has limited power resources. However, in many protocols, a node sends an interest packet by flooding, which consumes much power.

To solve this problem, a multipath content acquisition method has been proposed. In this method, since a requester node selects provider nodes, it does not need flooding for the transmission of an interest. However, relay nodes in one of the paths cache only half of the content and cannot send any content to the requester unless both parts of the content are cached.

In this paper, in order to increase the probability of caching the complete content, we propose a content acquisition method using overhearing. In this method, adjacent nodes of a relay node also receive content data. They cache the content data if another part of the content has been cached. As a result, the number of nodes that cache the complete content increases.
\end{abstract}

\section{Introduction}

Recently, a considerable number of studies have been devoted to ICN [1], a new paradigm for the future Internet. The basic idea of ICN is to change the Internet communication model by replacing host addresses with named contents. NDN [2] and CCN [3] are some wellknown current research projects in this area.

In a typical ICN, when a node requests a content, it simply broadcasts a request packet, called an interest, which includes the content name. A node that receives the interest and has cached the requested content sends corresponding content packets in response. Otherwise, it rebroadcasts the interest packet.

Although most works on ICN focus on wired environments, ICN has also been applied to wireless environments such as MANETs. Such MANETs are called information centric MANETs (IC-MANETs). MANETs are self-organized networks and can construct networks without any fixed infrastructure. In a MANET, every node can be connected directly or indirectly with other nodes by multihop communication. From these features, each node has limited resources.

Several content acquisition methods have been proposed for an IC-MANET. These methods include techniques of decreasing network traffic. For example, TOP-CCN [4] reduces the number of control packets by MPR-based packet flooding. E-CHANET [5] uses a counter-based suppression mechanism before packet transmissions. REMIF [6] reduces power consumption by restricting duplicate interest packet transmissions.

In these protocols, when some nodes have the same requested content, duplicate transmissions of the content occur. To solve this duplicate transmission, an informationcentric architecture using a mobile agent (MA method) [7] [8] has been proposed. This method applies a routing protocol using an MA [9] [10] to content acquisition. In this method, the MA manages location information and a content list of all nodes, and constructs a route from a provider node to a requester node. The MA method can realize content acquisition without any flooding of interest packets. Therefore, it can prevent duplicate transmissions. However, this method requires a technique of obtaining location information such as GPS. Therefore, it is difficult to use indoors. Moreover, it increases traffic owing to the use of control packets to manage the location information of the nodes.

To solve these problems, a multipath content acquisition method has been proposed [11]. If multiple nodes have the content requested by a requester node, the requester node selects two nodes as provider nodes, each of which sends a different half of the content. Therefore, no duplicate transmissions occur, and this method can reduce the network load and content acquisition delay. However, in this method, relay nodes in one of the content transmission paths cache only half of the content and cannot send any content to the requester unless both parts of the corresponding content are 
cached. In such a situation, the content cache is not used efficiently.

Therefore, in order to increase the probability of caching the complete content, we propose a content acquisition method using overhearing. In the proposed method, adjacent nodes of a relay node also receive content packets. They cache the content packets if another part of the content has been cached. As a result, the number of nodes that cache the complete content increases.

\section{Multipath Content Acquisition Method}

In this section, we describe the conventional multipath content acquisition method.

\subsection{Content request}

When a node requests a content, this node, called the requester node, sends an interest packet to the entire network by flooding. This packet includes a request of the content name. Also, it sets the pending timer. When a node that holds the requested content receives the interest packet, it replies with a content-holding packet to the requester node in order to inform it that it has the requested content. When the pending timer expires or the requester node receives the second content-holding packet, it sends a content request packet to the provider node(s), which is the source of the content-holding packet. The content request packet includes a requesting sequence number of the content. This enables split content transmission. If the requester node receives three or more content-holding packets, it ignores all but the first two. When the pending timer expires, if the requester node has received only one content-holding packet, the corresponding provider node sends all parts of the requested content.

A concrete example is shown in Fig. 1. In this figure, three nodes $\mathrm{A}, \mathrm{B}$ and $\mathrm{C}$ hold the requested content and receive an interest packet from the requester node. Then, these nodes send the content-holding packet to the requester. For example, if the arrival of the content-holding packets is in the order of nodes $\mathrm{A}, \mathrm{B}$ and $\mathrm{C}$, the packet from node $\mathrm{C}$ is ignored, and the requester node sends the content request packet to nodes $\mathrm{A}$ and $\mathrm{B}$.

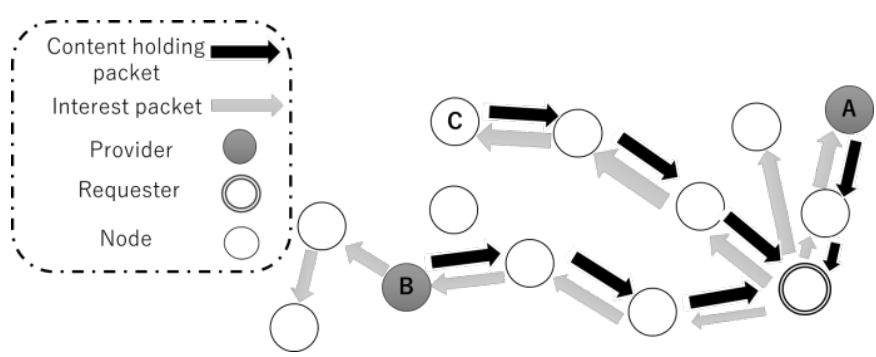

Figure 1: Content request

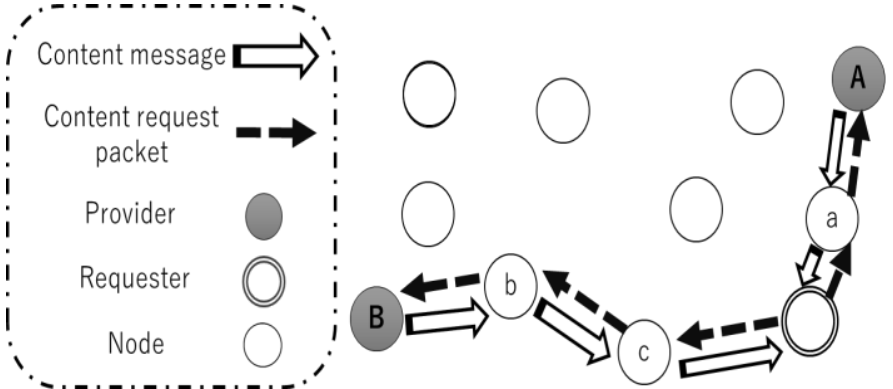

Figure 2: Content transmission and caching

\subsection{Content transmission and caching}

When the provider node receives the content request packet, it sends the requested content packet to the requester node by the route traced by the content request packet. At this time, the provider node transmits only the designated sequence section of the content. The relay nodes cache the sent part of the content. Only if a relay node can cache all parts of a content can return the corresponding contentholding packet as an interest packet of the content.

In Fig. 2, nodes A and B receive the content request packet from the requester node, and send the content packet. At that time, relay node ' $a$ ' caches one part of the content, and relay nodes ' $b$ ' and ' $c$ ' cache another part of the content. In this example, however, no relay node can cache the complete content.

\section{Proposed Method}

In this section, we describe the proposed method.

\subsection{Content request}

Similarly to the conventional method, when a node requests a content, it sends an interest packet to the entire network by flooding. When a node that holds the requested content receives the interest packet, it replies with a content-holding packet to the requester node and informs the requester node that it has the requested content. How the provider node is decided is also the same as that in the conventional method.

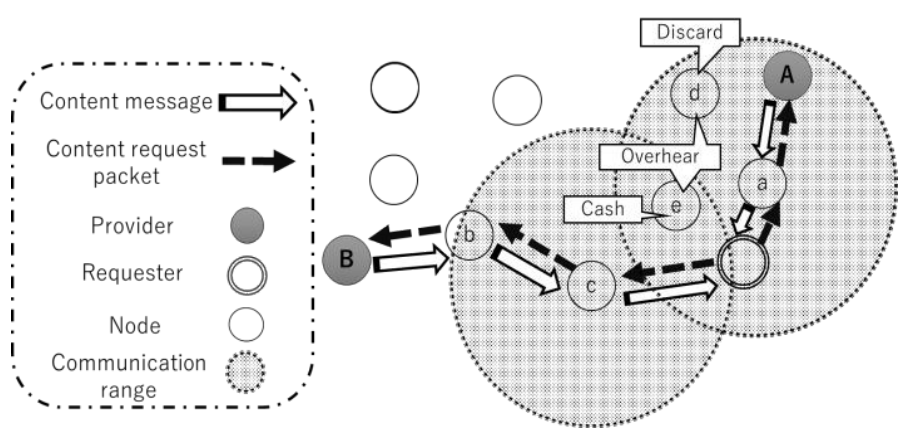

Figure 3: Content transmission and caching 


\subsection{Content transmission and caching}

When the provider node receives the content request packet, it sends the requested content packet to the requester node by the route traced by the content request packet. At this time, the provider node transmits only the designated sequence section of the content. The relay nodes cache the sent part of the content. The above process is the same as that in the conventional method. In addition to this process, the proposed method permits a node to overhear the content packets sent to its neighboring node. An overhearing node can also cache the overheard content packets. Moreover, in order to prevent the unnecessary cache of one part of a content, if a cached content is only half complete, it is discarded from the cache.

For example, in Fig. 3, nodes $\mathrm{A}$ and $\mathrm{B}$ receive the content request packet from the requester node, and send the requested part of the content packet. At that time, relay nodes ' $a$ ', ' $b$ ' and 'c' try to cache the content. However, they finally discard the cached part of the content because it is not complete. Suppose node 'd' is within the communication range of node ' $a$ ' and node ' $e$ ' is within the communication range of both nodes ' $a$ ' and ' $c$ '. In such a situation, these nodes can overhear the sent content packet from ' $a$ ' or ' $c$ ' and cache the received packet. Node ' $d$ ' finally discards the cached content because it is not complete. On the other hand, node ' $\mathrm{e}$ ' can cache both parts of the content. Therefore, it can send a content-holding packet if it receives the corresponding interest packet. By permitting overhearing and discarding an incomplete content, the proposed method can improve the efficiency of cache management.

\section{Computer Simulation}

The performance of the proposed method is evaluated by computer simulation to verify its effectiveness by comparison with the conventional method. Table 1 shows the simulation parameters.

Table 1: Simulation parameters

\begin{tabular}{|c|c|}
\hline $\begin{array}{c}\text { Simulation area [m] } \\
\text { (One side } \cdot \text { Square) }\end{array}$ & 1000 \\
\hline Simulation time [s] & 5000 \\
\hline $\begin{array}{c}\text { Communication range [m] } \\
\text { (Radius } \cdot \text { Circle) }\end{array}$ & 100 \\
\hline Number of nodes & 500 \\
\hline Cache size [contents] & $1,2,3,4,5$ \\
\hline Moving speed of node [m/s] & $1-9$ \\
\hline Content size [KB] & 500 \\
\hline Request rate [request/s] & 0.1 \\
\hline $\begin{array}{c}\text { Number of } \\
\text { content types [type] }\end{array}$ & 10,50 \\
\hline
\end{tabular}

In the simulation, all nodes are allocated within an area and randomly placed. During a simulation period, we randomly select a requester node and a request content type. The node sends content packets by UDP. The simulation area is a square of $1000[\mathrm{~m}]$. The communication range is $100[\mathrm{~m}]$. The number of nodes is 500. There are two different numbers of content types: 10 and 50 . To realize a situation where some contents are frequently requested, we set $p_{1}: p_{2}: p_{3}: \ldots: p_{N}=1: 2: 3: \ldots: N$, where $p_{i}$ is the probability that content $i$ is requested and $N$ is the number of content types. The size of each content is $500 \mathrm{kbytes}$. All nodes try to cache a copy of the content. When the cache is full, the oldest content is discarded. The evaluated factors are average power consumption, content reception rate and content reception delay.

\section{Simulation Results}

Figure 4 shows the average power consumption of each method. The power consumption of the proposed method is lower than that of the conventional method. In the proposed method, the number of nodes where the obtained content is complete increases. Therefore, the probability that a node with the requested content is near the requester node is larger than that in the conventional method. This means that the transmission hops become shorter. As a result, the power consumption decreases.

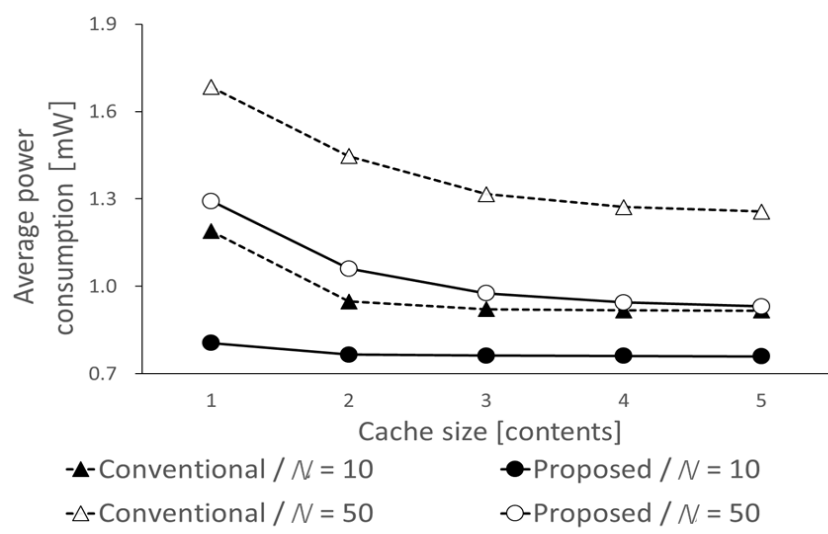

Figure 4: Average power consumption

Figure 5 shows the content reception rate, defined as the ratio of the number of contents for which at least one UDP packet arrives at a requester node to that of requested contents.

The figure shows that the rate is almost $100 \%$ regardless of the method and cache size used. 


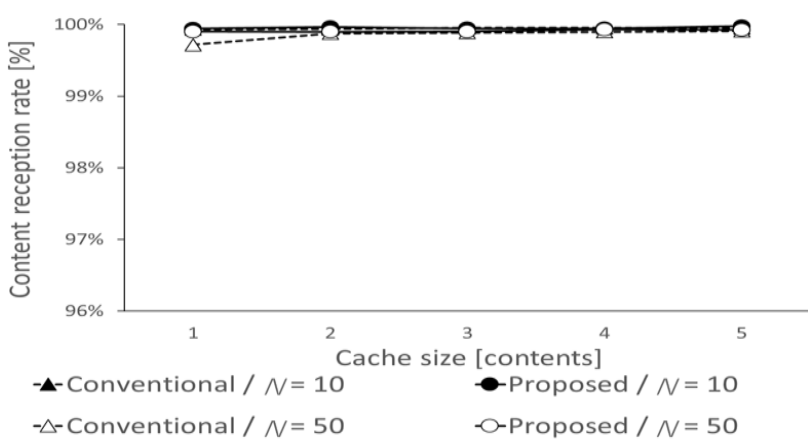

Figure 5: Content reception rate

Figure 6 shows the content reception delay, the time from the flooding of the interest packet to the acquisition of the content.

The figure shows that the proposed method has a shorter reception delay than the conventional method. In the proposed method, the content is cached in many nodes because of overhearing. Moreover, by discarding incomplete contents, the proposed method can cache contents more effectively than the conventional method. Therefore, the probability of a node with the requested content is higher than that in the conventional method. As a result, the reception delay decreases.

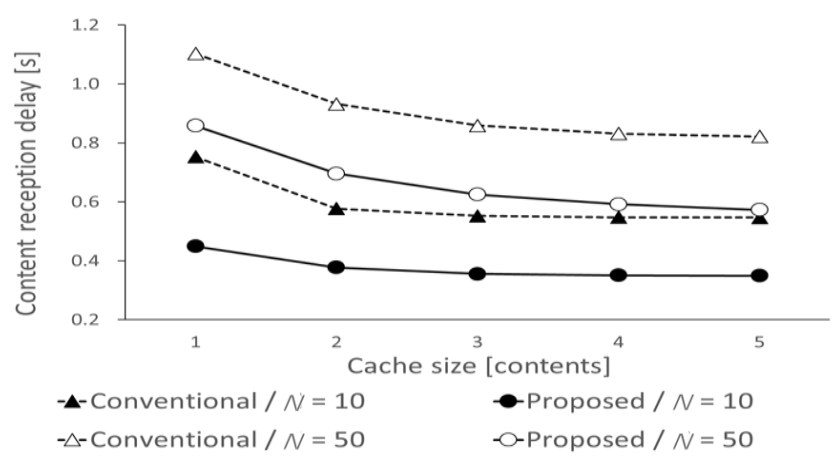

Figure 6: Content reception delay

We find from the simulation results that the average power consumption and content reception delay are improved compared with those observed by the conventional method. On the other hand, the content reception rate is almost the same. In the proposed method, the content is cached at many nodes by using overhearing. Also, by discarding incomplete contents, the proposed method can cache content more effectively than the conventional method. Therefore, the number of nodes that cache the complete content is increased. This indicates that the distance between a requester and a provider is shorter than that in the conventional method. As a result, the average power consumption and content reception delay are reduced while maintaining the content reception rate.

\section{Conclusions}

In this paper, we proposed a method of permitting a node other than relay nodes to cache contents using overhearing. Also, in the proposed method, if the cached content is not complete, it is discarded.

From the results of a computer simulation, we verify the effectiveness of the proposed protocol in terms of average power consumption and content reception delay.

\section{References}

[1] B. Ahlgren, C. Dannewitz, C. Imbrenda, D. Kutscher and B. Ohlman: A survey of information-centric networking, Communications Magazine, Vol. 50, No. 7, pp. 26-36, 2012.

[2] L. Zhang, A. Afanasyev, J. Burke, V. Jacobson, P. Crowley, C. Papadopoulos, L. Wang and B. Zhang: Named data networking, ACM SIGCOMM Computer Communication Review, Vol. 44, No. 3, pp. 66-73, 2014.

[3] V. Jacobson, D. K. Smetters, J. D. Thornton, M. F. Plass, N. H. Briggs and R. L. Braynard: Networking named content, Proceedings of the 5th International Conference on Emerging Networking Experiments and Technologies, ACM, pp. 1-12, 2009.

[4] J. Kim, D. Shin and Y.B. Ko: TOP-CCN: Topology aware content centric networking for mobile ad hoc networks, 19th IEEE International Conference on Networks (ICON), 2013.

[5] M. Amadeo, A. Molinaro and G. Ruggeri: E-CHANET: Routing, forwarding and transport in informationcentric multihop wireless networks, Computer Communications, Vol. 36, No. 7, pp. 792-803, 2013.

[6] R. A. Rehman, T. D. Hieu, H. Bae, S. Mah and B. Kim: Robust and efficient multipath interest forwarding for NDN-based MANETs, Wireless and Mobile Networking Conference, 2016.

[7] J. Fujikawa and S. Shiokawa: Information-centric architecture using mobile agent for MANET, Journal of Signal Processing, Vol. 22, No. 4, pp. 179-183, 2018.

[8] J. Fujikawa and S. Shiokawa: Improvement of information-centric architecture using mobile agent for MANET, IEICE Technical Report, Vol. 118, No. 282, pp.81-86, 2018.

[9] K. Tanaka and S. Shiokawa: Consideration of scalability for location based routing protocol using mobile agent in wireless multihop networks, NCSP' 13, pp.389-392, 2013.

[10] J. Fujikawa, F. Yamanobe and S. Shiokawa: Dual-path routing considering inter-path interference using a mobile agent in wireless multi-hop network, Transactions of IEICE, Vol. J100-B, No. 11, pp.912922, 2017. (in Japanese)

[11] J. Fujikawa and S. Shiokawa: Content acquisition method using multi-path for information-centric network, IEICE Technical Report, Vol. 119, No. 157, pp.19-22, 2019. 